\title{
THEILERIA MUTANS EN ROUMANIE
}

\author{
Par Tiberiu METZIANU
}

Au printemps de l'année 1946, nous avons été informé par le médecin vétérinaire ( $\mathrm{D}^{r}$ Popa) de la circonscription Iara (Turda) (Nord de la Transylvanie), que quelques bovidés (au nombre de neuf) avaient succombé avec une symptomatologie complexe et une évolution rapide. La maladie est apparue brusquement et les animaux succombent le premier ou le deuxième jour.

La température était élevée $\left(40^{\circ}-42^{\circ}\right)$, la respiration et la fréquence des battements du cœur accélérés, les muqueuses pâles.

Les animaux étaient abattus, sans appétit, avec amaigrissement avancé, la marche était incertaine, il y avait une diarrhée profuse avec stries de sang.

Des fragments des viscères, envoyés à notre laboratoire pour être examinés, nous ont permis de mettre en évidence une congestion de la muqueuse intestinale avec des ecchymoses et ulcérations. Les ensemencements d'organes (rate, foie) et les inoculations chez les animaux d'expérience ont été négatifs. Ne pouvant donner un diagnostic précis on a demandé au médecin-vétérinaire de signaler à temps les nouveaux cas, afun ae permettre de faire des recherches complémentaires.

Au printemps de l'année 1947 de nouveaux cas sont apparus avec la même symptomatologie. Le nombre des cas, cette fois-là, a été plus grand. D'après le complexe des symptômes observés, nous avons supposé l'existence d'une theileriose.

Les examens répétés du sang et du contenu ganglionnaire ont confirmé le diagnotic de Theileria mutans (1).

(1) Presque à la mème époque, nous avons diagnostiqué et publié pour la première fois en Roumanie des cas de theilériose par Theileria dispar dans le département de Cluj, en signalant des cas semblables dans la circonscription de lara. Dès le début, nous avons eu l'impression qu'il s'agissait, dans cette circonscription (Iara), de deux variétés de maladie. L'examen de laboratoire n'a confirmé ici que le diagnostic de Theileria mutans, dans le cas que nous avons pu étudier. Restent inexplicables les cas mortels avec ce diagnostic, fait qui nous porte à croire qu'il existe encore une variété dans cette région (la variété dispar) qui doit être recherchée l'année suivante.

Ans. de Pafasitologie, T, XXII, $\mathrm{N}^{\circ \mathrm{s}} 3-4,1947$, p. 137-142. 
La symptomatologie clinique des onze animaux observés par nous était assez réduite. Ils présentaient une forme subaiguë ou souvent une forme latente chronique.

La forme subaiguë de la maladie s'est manifestèe par : température élevée de $38^{\circ} 9-39^{\circ} 8$, même jusqu'à $40^{\circ}$, la pâleur de la muqueuse oculaire avec les yeux larmoyants. Diminution de l'appétit (anorexie) et de la rumination, atonie du rumen, diarrhée, quelquefois constipation, respiration un peu accélérée 20-30-60 par minute, pouls aussi accéléré, $80-90-100$ par minute. Ganglions préscapulaires et précruraux hypertrophiés arrivant jusqu'à la dimension d'un œuf. Les animeaux ont eu une tendance évidente à maigrir pendant l'évolution de la maladie.

La forme chronique, ou latente, de la maladie dans nos cas, s'est présentée avec un tableau symptomatologique suffisamment réduit. Les muqueuses oculaires pâles ou congestionnées avec une abondante sécrétion lacrymale.

La température a varié entre $38^{\circ}$ et $39^{\circ} 2$, avec une respiration normale ou un peu accélérée et sans modifications du cœur. Les ganglions préscapulaires et précruraux augmentés de volume.

Quelques animaux ont présenté seulement un accroissement des ganglions préscapulaires. Nous avons rencontré aussi des animaux qui, en apparence, ont donné l'impression d'être sains, mais chez lesquels nous avons mis en évidence le parasite dans le sang.

Par l'examen microscopique du sang nous avons trouvé, dans les hématies, "les gamétocytes 》 de Theileria mutans, et dans les cellules lymphatiques obtenues par ponctions ganglionnaires et de la rate, de rares «corps en grenade ». Nous n'avons exécuté les ponctions des ganglions préscapulaires qu'après leur découverte préalable par une incision des téguments.

Dans les frottis du sang, les gamétocytes de Theileria mutans ont présenté des morphologie différentes: formes elliptiques, rondes, en bâtons et anaplasmoïdes, surtout les formes elliptiques et rondes.

Les formes elliptiques (fig. 1-3) ont été les plus nombreuses (50$70 \%$, leurs dimensions varient entre : $2 \mu-2 \mu 5 ; 0 \mu 7-1 \mu$ ). Elles présentent une vacuole centrale; à la périphérie, le cytoplasme coloré en bleu et disposé à l'un des pôles de l'ellipse, tandis qu'au pôle opposé, on trouve la chromatine colorée en rouge.

Les formes rondes (fig. 2 et 4 ) ou circulaires ont un diamèfre de $1 \mu 1$ à $1 \mu 8$ avec une vacuole centrale. A la périphérie la chromatine, colonée en rouge, est disposée sur un demi-cercle, tandis que sur l'autre, nous trouvons le cytoplasme coloré en bleu, 
Les formes en bàton (fig. 5) sont représentées dans une proportion de 12 à 29 p. 100 . Elles présentent un noyau (point de chromatine) allongé ou rond qui occupe la moitié de la longueur du parasite et se continue par une queue de cytoplasme colorée en bleu. Leurs dimensions varient entre $2 \mu$ et $2 \mu 5$.

Les formes anaplasmoïdes, constituées par des grains de chromatine colorés en rouge, avec un diamètre de $0 \mu$ 8-1 $\mu$, sont disposées à la périphérie ou dans le centre du globule rouge, au nombre de une ou deux dans une hématie. Nous ne les avons mises en évidence que très rarement.

Les formes irrégulières (fig. 6) sont très rares et ont été observées dans les cas apyrétiques.

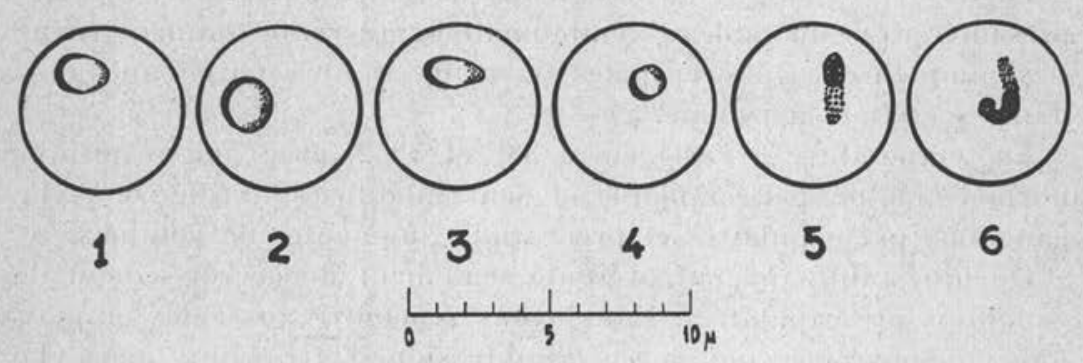

Fig. - Theileria mutans en Roumanie.

Le nombre des parasites, par rapport à celui des globules rouges, est en général très réduit dans nos cas, il atteignait 1 à 10 pour 2.000 hématies.

Dans la grande majorité des cas, nous avons trouvé un seul parasite dans le globule rouge, très rarement deux. Chez certains animaux, ils étaient altérés, présentant de la poïkilocytose et de l'anisocytose et même des corpuscules de Jolly.

Dans les cellules lymphatiques, à la suite des ponctions ganglionnaires et de la rate, nous avons trouvé des « corps en grenade intracellulaires de Theileria mutans. Ce sont des grains de chromatine colorés en rouge, irrégulièrement disposés d'un côté du cytoplasme, de dimensions qui varient entre $0 \mu 4$ et $1 \mu 8$ et de forme ronde ou un peu allongée. Leur nombre, dans une cellule, a été, dans nos cas, entre 2 et 15 . Les corps en grenade ont été mis en évidence en nombre très réduit, et chez quelques animaux ils n'ont pas été trouvés.

Le diagnostic différentiel doit être fait avec Theileria dispar ; il 
est souvent assez difficile. Cliniquement la symptomatologie de Theileria dispar est plus complexe, elle présente quelquefois une forme plus grave, mème mortelle.

Au point de vue parasitologique, chez Theileria mutans, le nombre de gamétocytes est réduit (5-20 pour 1.000 hématies). Les formes rondes et elliptiques sont en nombre réduit et de dimensions plus grandes, tandis que chez Theileria dispar elles sont plus petites et plus nombreuses.

L'accès parasitaire produit par T. mutans est plus long (quelques mois), par rapport avec celui de $T$. dispar qui est de quelques jours.

L'évolution de la maladie, dans les cas observés par nous, a été favorable, les animaux ont été guéris. Chez quelques-uns seulement l'affection est restée sous forme latente. En général on peut donc affirmer que le pronostic a été favorable.

Il y a tout de même un fait clinique curieux qui mérite d'être signalé (bien que le diagnostic microscopique de Theileria mutans ait été vérifié par des hommes compétents (Prof. Lavier et $\mathrm{D}^{x}$ Parrot), e'est la présence parmi les premiers cas parus en 1946 de 9 cas mortels.

Les tentatives thérapeutiques avec le bleu trypan, l'acaprine et la trypaflavine n'ont pas donné des résultats évidents par rapport aux cas qui n'ont pas été traités.

Si la thérapeutique de cette maladie n'a pas une si grande importance, on doit donner une grande attention à la prophylaxie, c'està-dire à l'enlèvement journalier des tiques de ces animaux. C'est le seul moyen de combattre cette maladie.

Nos recherches ont réussi à mettre en évidence, pour la première fois en Roumanie, la Theileria mutans, parasite originaire de l'Afrique du Nord, décrit par Theiler et Graaf et étudié par Ed. Sergent, Donatien, Parrot et Lestoquard.

La découverte de cette maladie, en Roumanie a une importance non seulement scientifique, mais aussi économique. Les recherches doivent être continuées pour voir si l'affection reste localisée seulement dans la région de Iara (Transylvanie) ou si elle a contaminé d'autres régions.

Tenant compte que cette maladie a été inconnue jusqu'à présent en Roumanie, et pour la confirmation de notre diagnostic, nous avons cru bien d'avoir l'opinion compétente des spécialistes étrangers, qui ont eu l'amabilité d'étudier nos lames et qui ont confirmé le diagnostic de theileriose causée par T. mutans. 
Nous remercions à cette occasion MM. le Prof. Lavier et le $\mathrm{D}^{r}$ Parrot pour leur amabilité et leur bienveillance.

\section{BiBLIOGRAPHIE}

Sergent (Ed.), Donatien (A.), Parkot (L.) et Lestoguand (F.) (in memoriam). Etudes sur les piroplasmoses bovines, Alger, 1945.

Schillivg et Meyer (F.). - Piroplasmosen, Handbuch pathogenen Mikroorgamen, 1927.

Doflein (F.) et Reichenow (Ed.). - Lehrbuch der Protozoenkunde, 1939.

Hutyra (Marek-Manninger). - Spezielle Pathologie und Therapie der Haustiere. Bиuмpt (E.). - Précis de Parasitologie, Paris, 1936.

Nevev-Lemaire (M.). - Traité de zoologie et parasilologie vétérinaire, 1936-1942. Inumoru (Gh.). - Sur les piroplasmes et piroplasmoses des bovidés en Transylvanie). Bul. Ass. Méd. Vét., Nr. 1-2, 1942.

- Sur les piroplasmes. Le cycle évolutif schizogonique (theileriose), Sibiu, 1945.

- La présence de theileriose en Roumanie, Cluj, 1947.

Metzianu (T.). - Theileria dispar en Roumanie, 1947.

Cluj (Roumanie). 